\title{
V Rechtspolitische Konsequenzen für eine Neugestaltung des Namensrechts
}

Was folgt aus dem bisherigen Befund rechtspolitisch? Mit dieser Frage stoße ich zum Kern meines Themas vor. Wenn ein Gesetzgeber die traditionellen Funktionen eines Rechtsinstituts (III.) aus übergeordneten Gründen nicht mehr vollständig umsetzen kann (IV.), dann muss der Gesetzgeber diese Funktionen als Regelungsmaximen aufgeben. Andernfalls kann der Gesetzgeber willkürliche Ergebnisse nicht vermeiden. Dass die Individualisierung und Klassifizierung des Namensträgers nicht mehr Richtschnur für die Ausgestaltung des Namensrechts sein sollte, ergibt sich aber auch losgelöst von diesen übergeordneten Gründen: Der Name taugt zur Erfüllung der traditionellen Funktionen zunehmend nicht mehr, auch weil diese Funktionen widersprüchliche Anforderungen an das Namensrecht stellen (oben III. 1.). Zudem sind die traditionellen Funktionen des Namens an sich rechtspolitisch zweifelhaft geworden, insbesondere die Klassifizierung der Bürger durch ihre Namen (oben IV. 1.).

\section{Besinnung auf die Selbstdarstellungsfunktion des Namens = Mehr Namenswahlfreiheit wagen}

Der Gesetzgeber sollte sich daher auf eine andere Funktion des Namens konzentrieren. Der Name dient als Teil unserer Persönlichkeit auch der Selbstdarstellung unserer Person in der Gesellschaft, ${ }^{107}$ die vor allem auf individuellen Assoziationen und Emotionen beruht, die ein bestimmter Name, sein sprachlicher Gehalt und Klang, auslöst. Über diese Selbstdarstellung durch seinen Namen sollte der Einzelne frei entscheiden dürfen, wie auch über andere Elemente des persönlichen Erscheinungsbilds in der Sozialsphäre, die menschlich beeinflussbar sind. Was unterscheidet den Namen - wenn man seine Individualisierungsund Klassifizierungsfunktion beiseitelässt - vom Bekleidungsstil oder sonstigen Habitus, den das Recht nur in Ausnahmefällen (Pflicht zur Uniform oder Amtstracht) vorgibt? Ein Namensrecht, das die Selbstdarstellungsfunktion des Namens ernst nimmt, muss die Bestimmung des Namens in erster Linie dem Namensträger

107 Hepting (Fn. 25) 2, 3; vgl. auch - insbesondere im Hinblick auf die Bedeutung des allgemeinen Persönlichkeitsrechts für den Namen (dazu bereits oben IV. 3.) - BVerfG 30.1.2002, BVerfGE 104, 373 = StAZ 2002, 72 = FamRZ 2002, 306 (308); BVerfG 18. 2. 2004, BVerfGE 109, 256 = StAZ 2004, 104 = FamRZ 2004, 515 (516); BVerfG 5.5.2009, BVerfGE 123, 90 = StAZ 2009, $179=$ FamRZ 2009, 939 (940, 942).

๖ OpenAccess. (๑) 2020 Juristische Gesellschaft zu Berlin, publiziert von De Gruyter.

(c) BY-NC-ND Dieses Werk ist lizenziert unter der Creative Commons Attribution-NonCommercial-NoDerivatives 4.0 License. https://doi.org/10.1515/9783110709872-006 
überlassen und eine umfassende Namenswahlfreiheit gewähren. ${ }^{108}$ Nicht die Freiheit des Einzelnen, seinen Namen zu wählen, ist danach begründungsbedürftig, sondern ihre Verweigerung oder Einschränkung.

Gegen eine solche Namenswahlfreiheit als Ausfluss der Selbstdarstellungsfunktion des Namens lassen sich zunächst keine privaten Interessen in Stellung bringen, wenigstens nicht im Grundsatz (zu Grenzen im Einzelfall sogleich V. 2. a und b). Zwar wird der Namensträger seinen Namen auch anhand der im Namen traditionell kodierten Eigenschaften (vgl. oben III. 1.) wählen. Diese werden oftmals die für die Namenswahl maßgeblichen Assoziationen und Emotionen auslösen. Anders als bei der Klassifizierungsfunktion entscheidet bei der Selbstdarstellungsfunktion damit der Namensträger selbst, welche Eigenschaften zutreffend oder nicht - sein Name transportieren könnte, und nicht der Staat, welchen Namen der Namensträger aufgrund seiner Eigenschaften zu tragen hat. Eine relevante Irreführung der anderen Mitglieder der Gesellschaft über die im Namen kodierten Eigenschaften ist allerdings nicht zu befürchten. Die Zeiten sind vorbei, in denen im gesellschaftlichen oder gar geschäftlichen Verkehr der Name einer Person andere Gesellschaftsmitglieder zu Dispositionen veranlasst hat, weil diese darauf vertrauen, dass die im Namen kodierten Eigenschaften in der Person des Namensträgers auch zutreffen, etwa dieser einer bestimmten Familie angehört oder eine bestimmte gesellschaftliche Stellung einnimmt. Jedenfalls insoweit gilt - jenseits einer Täuschung über die Identität - heute uneingeschränkt: Der

108 Siehe etwa bereits Sturm (Fn. 3) 618 f. (Forderung nach einer „größtmöglichen Wahlfreiheit“); Sacksofsky (Fn. 77) 375 (Forderung nach Verzicht „auf die Überregulierung im Namensrecht“); Andrea Woelke Namensrecht und Selbstbestimmung, FamRZ 2004, 1342 (für eine freie Namenswahl); sogar aus verfassungsrechtlicher Perspektive für eine grundsätzliche Verfügungsbefugnis des Einzelnen über seinen Namen auch Alexander Blankenagel Das Recht, ein „Anderer“ zu sein, DÖV 1985, 953 (961 ff.); vgl. auch Michael Grünberger Von Bernhard Markus Antoinette zu Anderson Bernd Peter - Von der Ordnungsfunktion und der Identitätsfunktion des Vornamens, AcP 207 (2007) 314 (338) (jedenfalls Namenswahlfreiheit im Hinblick auf den Vornamen); Christian Majer Aus Ahmed Mustafa wird Albert Mustermann - Eindeutschung des Namens jenseits der kollisionsrechtlichen Namensangleichung nach Art. 47 EGBGB, StAZ 2018, 80 (für die Wahlmöglichkeit zugunsten eines deutschen Namens; hierfür auch Matthias Hettich Rezension von Mertens, Das Namensänderungsgesetz [2018] StAZ 2018, 294 [295]); für eine Liberalisierung der öffentlichrechtlichen Namensänderung Laura Antonia Mertens Das Namensänderungsgesetz (2018) S. 189 ff.; dies. Über Sinn und Unsinn der öffentlich-rechtlichen Namensänderung - sieben Fragen zum NamÄndG, StAZ 2018, 329 (331 f.); Hettich aaO 295. Allgemein für eine Liberalisierung des Namensrechts auch die Eckpunkte zur Reform des Namensrechts (Fn. 2) S. 4 f. unter II. 3. 
Name ist „Schall und Rauch“109. Hinzu kommt, dass das Recht heute ohnehin im privaten Verkehr Pseudonyme gestattet und grundsätzlich keine Pflicht zur Führung des rechtlichen Namens besteht (näher noch unten VI.).

Aber auch öffentliche Belange stehen einer Namenswahlfreiheit nicht prinzipiell (für Einzelfälle noch unten V. 2. c und d) entgegen. Insbesondere dürfte ein Mehr an Namenswahlfreiheit die Arbeit der Sicherheitsbehörden nicht beeinträchtigen. $\mathrm{Zu}$ berücksichtigen ist, dass es bereits nach geltendem Recht häufig zu Namensänderungen kommt, welche die Sicherheitsbehörden nicht vor unlösbare Aufgaben stellen. Womöglich nimmt die Anzahl der Namensänderungen nach Einführung einer Namenswahlfreiheit sogar ab. Denn diese beinhaltet auch die negative Wahlfreiheit, den Namen nicht in Konstellationen $\mathrm{zu}$ ändern, in denen das Recht dies heute von Gesetzes wegen tut (siehe unten V. 2. a). Die Tatsache, dass die Individualisierung der Bürger für den Staat in Zeiten der Digitalisierung effektiver über Personennummern erfolgt, wurde bereits angesprochen (oben III. 1.). Auch Mitteilungspflichten der Standesämter und die zentrale Registrierung von Namensänderungen räumen Bedenken der öffentlichen Sicherheit gegen eine Namenswahlfreiheit aus, ${ }^{110}$ jedenfalls in den wenigen Bereichen, in denen die Führung des rechtlichen Namens überhaupt für den Bürger verpflichtend ist (erneut näher unten VI.).

Entscheidend ist auch ein weiterer Punkt, der für eine Konzentration auf die Selbstdarstellungsfunktion des Namens und eine grundsätzlich umfassende Namenswahlfreiheit streitet. Die übergeordneten Gründe, die den Gesetzgeber zur Relativierung der traditionellen Namensfunktionen gezwungen haben (oben IV.), stehen einer Namenswahlfreiheit des Namensträgers nicht entgegen. Im Gegenteil: Eine Namenswahlfreiheit würde der Gleichheit vor dem Gesetz, dem Elternrecht und dem allgemeinen Persönlichkeitsrecht, aber auch der namensrechtlichen Integration sowie der Personenfreizügigkeit und dem Diskriminierungsverbot sogar einen breiteren Raum verschaffen, als das bisher der Fall ist, wo der Gesetzgeber bislang lediglich punktuell die traditionellen Namensfunktionen relativiert hat. ${ }^{111}$ Eine Stärkung der Selbstdarstellungsfunktion des Namens war deshalb oftmals Reflex bei der gesetzgeberischen Relativierung der namensrechtlichen Individualisierung und Klassifizierung, ${ }^{112}$ aber eben nur insoweit, als

109 Zur wirklichen Bedeutung der Antwort auf die Gretchenfrage etwa Walter Pintens Ist der Name Schall und Rauch? in: Diethelm Klippel/Hans-Jürgen Becker/Reinhard Zimmermann (Hrsg.) Colloquia für Dieter Schwab zum 65. Geburtstag (2000) S. 37 (37 f.).

110 So auch bereits Blankenagel (Fn. 108) 962; vgl. auch Eckpunkte zur Reform des Namensrechts (Fn. 2) S. 8 unter III. 11.

111 In diese Richtung auch Lettmaier (Fn. 74) 6.

112 Vgl. Hepting (Fn. 16) 119. 
es aus den übergeordneten Gründen (oben IV. 2-5.) erforderlich war. Auch die zahlreichen Wertungswidersprüche im geltenden Namensrecht würde eine solche Namenswahlfreiheit auflösen, die allesamt aus einer Beschränkung der namensrechtlichen Privatautonomie resultieren, um die traditionellen Funktionen des Namens, soweit wie noch möglich (oben IV.), umzusetzen. So schrieb Thomas Wagenitz - der im Bundesjustizministerium und später am Bundesgerichtshof das Namensrecht lange begleitet hat - nach einer der vergangenen Namensrechtsreformen im Jahr 1998 mit etwas Resignation: „Kein Wunder, daß der Ruf nach weiteren Reformen nicht verstummt [...]. Vielleicht wird er das Namensrecht bis zur völligen Freigabe jeder Namenswahl durch einen - uns wohl fernen Gesetzgeber begleiten."

Eine Liberalisierung des Namensrechts wäre schließlich auch aus historischvergleichender Perspektive konsequent. Mit der Einführung einer Namenswahlfreiheit des Namensträgers würde sich das deutsche Recht aktuellen Entwicklungen in benachbarten Rechtsordnungen anschließen (näher noch unten V. 3.) und auch rechtsgeschichtlich würde sich ein Kreis schließen: Wie bereits angedeutet (oben III. 1.) und noch näher zu sehen sein wird (unten VI.), war die Bestimmung des Namens erst spät ein Thema für das Recht. Des Weiteren würde eine Namenswahlfreiheit das Namensrecht an die heutigen Wertungen eines anderen Rechtsgebiets annähern, das historisch eng mit dem Namensrecht verknüpft ist: das Firmenrecht, ${ }^{114}$ das bereits vor mehr als 20 Jahren eine starke Liberalisierung erfahren hat. ${ }^{115}$ Damals hat der Gesetzgeber ebenfalls die Klassifizierungsfunktion der handelsrechtlichen Firma aufgegeben und neben der Personen- und Sachfirma auch die Phantasiefirma zugelassen, soweit diese nach $\S 18$ des Handelsgesetzbuchs zur Kennzeichnung geeignet ist, Unterscheidungskraft besitzt und das Irreführungsverbot wahrt. Ähnlich wie hier für das Namensrecht vorgeschlagen, war es erklärtes Ziel der Firmenrechtsreform, die Selbstdarstellungsfunktion der Firma zu stärken und Unternehmen ,größere Wahlfreiheit bei der Bildung aussagekräftiger und werbewirksamer Firmen“ zu geben. ${ }^{116}$ Im Firmenrecht ist der Schutz der Gesellschaftsmitglieder und speziell

113 Thomas Wagenitz Neues Recht in alten Formen: Zum Wandel des Kindesnamensrechts, FamRZ 1998, 1545 (1552).

$114 \mathrm{Zu}$ historischen Parallelen bei der Entwicklung des Firmen- und Namens(schutz)rechts Klippel (Fn. 32).

115 Mit dem Gesetz zur Neuregelung des Kaufmanns- und Firmenrechts und zur Änderung anderer handels- und gesellschaftsrechtlicher Vorschriften vom 22.6.1998, BGBl. 1998 I 1474.

116 Begründung des Entwurfs eines Gesetzes zur Neuregelung des Kaufmanns- und Firmenrechts und zur Änderung anderer handels- und gesellschaftsrechtlicher Vorschriften, BT-Drucks. 13/8444, S. 35. 
der Verbraucher vor einer Irreführung über den Gegenstand des Unternehmens durch eine „unrichtige“ Firma sehr viel bedeutsamer als im Namensrecht. Zudem besteht hier anders als beim rechtlichen Namen (unten VI.) sogar eine Pflicht des Unternehmers, die handelsrechtliche Firma zu führen. ${ }^{117}$ Daher drängt sich der Schluss auf: Wenn das Firmenrecht eine weitreichende Wahlfreiheit gewährt, darf das Namensrecht nicht hinterherhinken.

\section{Notwendige Grenzen der Namenswahlfreiheit}

Freilich dürfte diese Namenswahlfreiheit nicht unbegrenzt sein und der Name nicht gänzlich den sich dann wieder herausbildenden Sitten und Gebräuchen (vgl. auch noch näher unten VI.) überlassen werden. Wie allgemein bei der Privatautonomie muss auch im Namensrecht gelten: Keine Freiheit ohne Grenzen.

\section{a) Wohl des Namensträgers, insbesondere Kindeswohl bei der Namensbestimmung durch die Eltern}

Eine erste Schranke muss das Recht immer dann aufstellen, wenn Dritte den Namen für den Namensträger festlegen, vor allem wenn die Eltern in Ausübung ihrer Personensorge (vgl. bereits oben I.) den Namen ihres Kindes erstmalig wählen. Hier darf die Wahl des Namens - wie alle Entscheidungen im Rahmen von Fürsorgeverhältnissen - nicht dem Wohl des Namensträgers widersprechen, also insbesondere nicht dem Kindeswohl. Ein mutiger Gesetzgeber würde es bei einem solchen allgemeinen Kindeswohlvorbehalt belassen und darauf vertrauen, dass die Standesämter, Personenstands- und Familiengerichte - wie derzeit bereits beim Vornamen (oben I.) - das namensrechtliche Wohl des Namensträgers ausreichend schützen. Ein solcher Kindeswohlvorbehalt würde auch für hinreichende Flexibilität sorgen. Denn selbstverständlich ist das namensrechtliche Kindeswohl nicht nur einzelfallbezogen im Hinblick auf die familiäre und gesellschaftliche Umgebung des Kindes zu bestimmen, sondern auch dynamisch ausgestaltet und kann sich - je nach den namensbezogenen Sitten und Bräuchen - wandeln.

Die Wahl welcher Namen durch die Eltern für das Kind würde man aus heutiger Sicht als kindeswohlwidrig ausschließen? (1) Unzulässig, weil kindeswohlwidrig, dürfte die Wahl eines Familiennamens sein, der keinerlei Bezug zum

117 BayObLG 20.9.1967, BayObLGZ 1967, 353 (355). 
familiären Umfeld des Kindes aufweist. In den derzeitigen gesellschaftlichen Verhältnissen dokumentiert der Familienname eine Verbindung zu den Eltern und deren Familien. Die Klassifizierungsfunktion kann subjektiv für Kinder notwendiges Element des Kindeswohls sein. ${ }^{118}$ (2) Ebenfalls kindeswohlwidrig dürfte ein Familienname sein, der ohne näheren Grund von dem Namen der Vollgeschwister abweicht (derzeit § 1617 Abs. 1 S. 3 BGB), jedenfalls wenn man die Geschwisterbindung gerade bei Krisen in der Elternbeziehung als Stabilitätsanker sieht und den gemeinsamen Familiennamen als Beitrag zur Verfestigung der Geschwisterbindung. ${ }^{119}$ (3) Schließlich dürfte auch eine spätere Änderung des Kindesnamens durch die Inhaber der Personensorge dem Kindeswohl im Regelfall widersprechen. Wie bereits Michael Coester vor vielen Jahren in seiner Göttinger Antrittsvorlesung dargelegt hat, sind - die speziell im geltenden Namensrecht oftmals allzu leichtfertig vorgesehenen - Namenswechsel des Kindes ${ }^{120}$ problematisch: Für das Kind kann ein „kontinuierlicher Name [...] gerade bei wechselnden Familienstrukturen der hilfreiche Strohhalm sein, an dem das Kind seine unaustauschbare Identität bei sonst fluktuierenden Bezugsverhältnissen festmacht. Wechselt sein Name mit jeder Veränderung auf Elternseite, wird es ihm schwerer fallen, sich als eigenständiges Individuum unabhängig von den Personen zu verstehen, denen es rechtlich oder sozial zugeordnet ist.“" ${ }^{121}$ Diesen Worten ist nichts hinzuzufügen. Bedauerlicherweise hat sich allerdings der deutsche Gesetzgeber bisher der Erkenntnis verschlossen, dass Namensänderungen des Kindes, speziell durch nachträgliche Wahlmöglichkeiten der Eltern und durch Erstreckung von Namensänderungen der Eltern, aus Sicht des Kindeswohls bedenklich sind. Zwar bezieht das geltende Namensrecht das Kind in eine Namensänderung durch die Eltern regelmäßig ab Vollendung des fünften Lebens-

118 Das deutet auch das BVerfG 30.1.2002, BVerfGE 104, $373=$ StAZ 2002, 72 = FamRZ 2002, 306 (310) an, wonach der Name dem Kind verhelfe, ,eine Identität zu finden und Individualität zu entwickeln. Namenlos kann das Kind nur schwerlich eigene Persönlichkeit entfalten und eine Beziehung zu anderen aufbauen. Vom Persönlichkeitsrecht des Kindes umfasst ist deshalb auch das Recht auf Namenserhalt als wesentliche Voraussetzung für die Entwicklung seiner Persönlichkeit. Dies betrifft den Vornamen wie den Familiennamen. Sieht die Rechtsordnung die Familiennamensführung vor, so ist dieser Name das Mittel, mit Hilfe dessen sich das Kind in ein Verhältnis zu anderen setzen lernt“.Vgl. auch Lettmaier (Fn. 74) 7, die deshalb rechtspolitisch eine Namenswahlfreiheit ablehnt, was meines Erachtens zu weit geht, wenn man - wie hier vorgeschlagen - diese unter einen Kindeswohlvorbehalt stellt.

119 Anders Schwenzer (Fn. 43) 396, die sich für unterschiedliche Namen von Geschwistern „durchaus anerkennenswerte Motive“ vorstellen kann.

120 Siehe die Zusammenstellung bei Hepting/Dutta (Fn. 6) Rn. V-431 ff. und Rn. V-581 ff.

121 Coester (Fn. 43) 302. 
jahres ein. ${ }^{122}$ Diese formelle Einbeziehung garantiert aber nicht die Kindeswohlgemäßheit der Namensänderung, solange der Inhaber der elterlichen Sorge das Kind vertritt oder das meist ab Vollendung seines 14. Lebensjahres selbständig entscheidende Kind sich kaum gegen den Namensänderungswunsch der Eltern stellen wird. Eine Namenswahlfreiheit der Eltern mit Kindeswohlvorbehalt würde das namensrechtliche Kindeswohl sehr viel besser verwirklichen als die schematischen Namenswechselnormen der lex lata. ${ }^{123}$

Natürlich müssten bei der Namenswahlfreiheit der Eltern für ihr Kind auch eine ganze Reihe weiterer Fragen geregelt werden: Welchen Namen erhält das Kind, wenn die Eltern sich nicht einigen? Hier könnte der Gesetzgeber ohne Weiteres auf die Regeln zurückgreifen, die zum Zuge kommen, wenn die Eltern bei der Ausübung der elterlichen Sorge uneinig sind, bei uns also allgemein auf $\S 1628$ BGB oder speziell auf den derzeitigen $\S 1617$ Abs. 2 und 3 BGB. ${ }^{124}$ Muss eine Person, deren Name von einer Wahl des Familiennamens des Kindes betroffen ist, weil dieser geändert oder gewählt wird, der Namenswahl zustimmen? Diese Frage ist grundsätzlich $\mathrm{zu}$ bejahen, ${ }^{125}$ jedenfalls für einen nicht sorgeberechtigten Elternteil oder einen Stiefelternteil. Ab welchem Alter muss das Kind bei der Namensbestimmung einbezogen werden oder kann ohne Zustimmung der Eltern seinen Namen wählen? Richtigerweise sollte ein Minderjähriger ab Vollendung des 16. Lebensjahrs über seinen Namen allein und ab Vollendung des 14. Lebensjahrs mit Zustimmung seines gesetzlichen Vertreters (vgl. etwa § 45a Abs. 2 PStG) entscheiden dürfen, ${ }^{126}$ wobei auch hier - nach dem im vorigen Absatz Gesagten - ein Kindeswohlvorbehalt gelten sollte.

Ein voraussetzungsloses Namensänderungsrecht würde im Übrigen dazu führen, dass der Namensträger sein namensrechtliches Wohl stets aktualisieren

122 Etwa in den Fällen des § 1617a Abs. 2 S. 2 Fall 2, § 1617b Abs. 1 S. 3 und Abs. 2 S. 1, § 1617c Abs. 1 S. 1 und Abs. 2, § 1618 S. 3 Fall 2, § 1757 Abs. 2 S. 2 und Abs. 3 S. 2 BGB. Vgl. aber auch $\S 4$ NamÄndG, der automatisch und ohne Einwilligung der Kinder die öffentlichrechtliche Namensänderung auf Kinder des Namensträgers erstreckt, wenn die Kinder bislang den Namen des Namensträgers getragen haben und für die Kinder die elterliche Sorge des Namensträgers besteht. Die Verwaltungspraxis der Namensänderungsbehörden macht allerdings, soweit ersichtlich, überwiegend von dem in $\S 4$ NamÄndG vorgesehenen Vorbehalt Gebrauch und schließt eine Erstreckung der Namensänderung auf die Kinder aus; näher hierzu Hepting/Dutta (Fn. 6) Rn. V$906 \mathrm{f}$.

123 Zu Recht spricht Hilbig-Lugani (Fn. 20) § 1617a BGB Rn. 22 von einer „Erosion der Namensstabilität“ im Kindesnamensrecht, nach der „eher die Anpassung des Familiennamens an die wechselnden Lebensverhältnisse als Grundsatz erscheint“.

124 Vgl. auch die Eckpunkte zur Reform des Namensrechts (Fn. 2) S. 5 unter III. 3.

125 So auch die Eckpunkte zur Reform des Namensrechts (Fn. 2) S. 7 unter III. 5.

126 Siehe auch die Eckpunkte zur Reform des Namensrechts (Fn. 2) S. 6 unter III. 4 b. 
kann. Insbesondere könnte der Namensträger Entscheidungen seiner Eltern zur Namenswahl revidieren. Das betrifft speziell auch den Vornamen, ${ }^{127}$ über dessen Wahl - wie Studien zeigen ${ }^{128}$ - oftmals ästhetische Präferenzen der Eltern entscheiden. Die Ästhetik des Vornamens hängt damit nicht nur von Moden ab, die sich ändern, sondern liegt bereits allgemein im Auge des Betrachters. Ob die Namensträger die ästhetischen Präferenzen der Eltern teilen, sollten sie ab Vollendung des 16. Lebensjahrs selbst entscheiden können.

\section{b) Schutz seltener Familiennamen}

Im Rahmen einer zweiten Schranke der Namenswahlfreiheit könnte der Gesetzgeber auch über einen Schutz seltener Familiennamen nachdenken. ${ }^{129}$ Ähnlich wie im dänischen und schwedischen Recht - die bereits heute eine freie Namenswahl des Namensträgers zulassen ${ }^{130}$ - könnte der Gesetzgeber vorsehen, dass nur Namen wählbar sind, die eine bestimmte Verbreitung besitzen, die etwa, wie in Dänemark und Schweden, mindestens 2.000 Menschen rechtmäßig führen. ${ }^{131}$

Eine solche Grenze der Namenswahlfreiheit würde ein subjektives Klassifizierungsempfinden der Träger seltener Namen schützen, die nach außen sichtbar alleine der betreffenden generationenübergreifenden Gruppe angehören wollen. ${ }^{132} \mathrm{Ob}$ die Interessen dieser Namensträger schützenswert sind, steht auf einem anderen Blatt. Ein Staat, der wie die Bundesrepublik in Art. 3 Abs. 3 S. 1 GG eine Benachteiligung oder Bevorzugung wegen der „Herkunft“ verbietet, worunter die „ständisch-soziale Abstammung und Verwurzelung“ $\mathrm{zu}$ verstehen ist, ${ }^{133}$ sollte keine Rücksicht auf dynastische Befindlichkeiten einzelner Namensträger nehmen. Vor Eingriffen in das subjektive Namensrecht schützt $§ 12$ BGB ausreichend. Hinzu kommen zahlreiche weitere Schwierigkeiten, die mit einem solchen Raritätenschutz verbunden wären, neben der statistischen Erhebung der Verbreitung bestimmter Namen insbesondere zwangsläufig willkürliche Grenzziehungen

127 Grünberger (Fn. 108) 338.

128 Siehe etwa Frauke Rüdenbusch Motive der Vornamenswahl, StAZ 2014, 323.

129 Angedacht auch von Blankenagel (Fn. 108) 962 (Schutz von Familiennamen in ,überschaubaren Gruppen“).

130 Siehe im Hinblick auf den Nachnamen $\S \S 1$ ff. des dänischen Navneloven; $\S \S 13$ ff. des schwedischen Lag om personnamn.

$131 \S 3$ Abs. 1 des dänischen Navneloven; §16 Abs. 3 des schwedischen Lag om personnamn. 132 In diese Richtung etwa von Hein (Fn. 94) 521.

133 BVerfG 25.5.1956, BVerfGE 5, 17 = FamRZ 1956, 217 (218). 
durch den Gesetzgeber: Ab welcher Anzahl von Namensträgern ist ein Familienname nicht mehr selten? Wie sind neu gebildete Namen zu behandeln, die bisher noch überhaupt nicht verbreitet sind? Weshalb soll der Gesetzgeber einen Namen, der wie „Dutta“ in Deutschland recht selten ist, vor einer Namenswahl durch Dritte schützen, wenn dieser Name in Indien und konkret in Westbengalen sehr verbreitet ist?

\section{c) Länge des Namens}

Auch im Hinblick auf die Länge des Namens sollte man der Namenswahlfreiheit keine Grenzen setzen, jedenfalls solange eine staatliche Registrierung oder Beurkundung nicht an die Grenzen stößt und zwingende Ordnungsinteressen des Staates gewahrt sind (vgl. bereits oben III. 2.). Ohnehin hat der Gerichtshof der Europäischen Union in seiner namensrechtlichen Rechtsprechung (oben IV. 5.) festgehalten, dass die Mitgliedstaaten die Anerkennung eines in einem anderen Mitgliedstaat erworbenen zusammengesetzten oder langen Namens nicht unter Verweis auf praktische Gründe oder auf Erwägungen der Verwaltungsvereinfachung verweigern dürfen. ${ }^{134}$ Wenn man das Namensrecht bereits rechtspolitisch vom Kopf auf die Füße stellt und die Namenswahlfreiheit in das Zentrum rückt, dann sollte diese Freiheit einheitlichen Grenzen unterliegen und nicht außerhalb des unionsrechtlichen Anerkennungsgebots enger gefasst sein.

\section{d) Öffentliche Ordnung}

Jedenfalls wird die öffentliche Ordnung die Namenswahlfreiheit begrenzen müssen, ${ }^{135}$ wie etwa derzeit der ordre-public-Vorbehalt beim Namenswahlrecht nach Art. 48 EGBGB. Der Staat sollte nicht verpflichtet sein, sittenwidrige oder anstößige Namen in seine Personenstandsregister einzutragen. ${ }^{136}$ Auch der Gerichtshof der Europäischen Union hat den ordre public der Mitgliedstaaten als Grenze des namensrechtlichen Anerkennungsgebots (oben IV. 5.) mehrfach an-

134 EuGH 14.10.2008, Rs. C-353/06 (Stefan Grunkin und Dorothee Regina Paul) Slg. 2008, I-7639 = StAZ 2009, 9 = FamRZ 2008, 2089, Rn. 36; EuGH 2.6.2016, Rs. C-438/14 (Nabiel Peter Bogendorff von Wolffersdorff gegen Standesamt der Stadt Karlsruhe und Zentraler Juristischer Dienst der Stadt Karlsruhe) StAZ 2016, 203 = FamRZ 2016, 1239, Rn. 60.

135 Vgl. auch die Eckpunkte zur Reform des Namensrechts (Fn. 2) S. 7 unter III. 6. 136 Vgl. auch die Eckpunkte zur Reform des Namensrechts (Fn. 2) S. 7 unter III. 6. 
erkannt. ${ }^{137}$ Zur öffentlichen Ordnung wird man auch zählen müssen, dass der gewählte Name eine Namensqualität besitzt, ${ }^{138}$ was man - jedenfalls derzeit - bei Zahlen und Symbolen verneinen müsste. Insoweit erfüllt der Name eine „Kommunikationsfunktion im Alltag“, indem er „den Einzelnen ansprech- und beschreibbar“ macht. ${ }^{139}$ Auch die Grundstrukturen des Namens, die heute in Deutschland mindestens aus einem Vor- und Familiennamen bestehen, ${ }^{140}$ wird man zur öffentlichen Ordnung zu zählen haben. ${ }^{141}$ Selbstverständlich würde sich nach einer Einführung einer Namenswahlfreiheit die öffentliche Ordnung - die wie das Kindeswohl (oben V. 2. a) dynamisch ist - langfristig an die namensbezogenen Sitten und Gebräuche anpassen.

Allerdings sollte die Wahl eines Namens mit so genannten „Scheinadelstiteln“ nicht gegen die öffentliche Ordnung verstoßen. „Scheinadlige“ haben in den vergangenen Jahren immer wieder die Gerichte - bis zum Bundesgerichtshof ${ }^{142}$ und zum Gerichtshof der Europäischen Union ${ }^{143}$ - mit namensrechtlichen Fragen beschäftigt. Vor allem ging es hier um die Frage, ob Namen, die in anderen Mitgliedstaaten um ehemalige Adelsbezeichnungen angereichert wurden, im Rahmen der bereits skizzierten Rechtsprechung zum unionsrechtlichen Anerkennungsgebot (oben IV. 5.) anzuerkennen sind und nach Art. 48 EGBGB gewählt werden können. Freilich gibt es die Kategorie der „Scheinadligen“ rechtlich anders etwa als den Scheinkaufmann - eigentlich gar nicht. ${ }^{144}$ Nach Art. 109

137 EuGH 22.12.2010, Rs. C-208/09 (Ilonka Sayn-Wittgenstein gegen Landeshauptmann von Wien) Slg. 2010, I-13693 = StAZ 2011, 77 = FamRZ 2011, 1486; EuGH 2.6.2016, Rs. C-438/14 (Nabiel Peter Bogendorff von Wolffersdorff gegen Standesamt der Stadt Karlsruhe und Zentraler Juristischer Dienst der Stadt Karlsruhe) StAZ 2016, 203 = FamRZ 2016, 1239.

138 Vgl. auch die Eckpunkte zur Reform des Namensrechts (Fn. 2) S. 7 unter III. 6.

$139 \mathrm{Zu}$ Recht Lettmaier (Fn. 74) 7, deren Folgerung, deshalb eine Namenswahlfreiheit zu verweigern, aber auch insoweit (vgl. bereits oben Fn. 118) meines Erachtens überzogen ist.

140 Vgl. auch BGH 19.2.2014, StAZ 2014, 139 = FamRZ 2014, 741 (743): „Der nach deutschem Recht gebildete bürgerliche Name einer natürlichen Person enthält zwingend einen Namensteil, der mit der Übertragbarkeit auf den Ehegatten und die Kinder auch die Aufgabe des Familiennamens erfüllen kann und einen anderen Namensteil, der als Vorname die Mitglieder einer Familie und allgemein die Träger des gleichen Familiennamens voneinander unterscheidbar macht“, wobei ein Vatersname nach Ansicht des BGH unschädlich ist.

141 Auch bisher wurde losgelöst von Art. 47 EGBGB bei einem Statutenwechsel zum deutschen Recht ein vor dem Wechsel nach ausländischem Recht erworbener Name auch ohne Angleichungserklärung des Namensträgers angeglichen, ausführlich Dennis Solomon Objektive Angleichung nach Eingangsstatutenwechsel, StAZ 2018, 265.

142 BGH 14.11.2018, StAZ 2019, 77 = FamRZ 2019, 218.

143 Nachweise oben in Fn. 134.

144 Martin Otto „Scheinadliger“ Namenserwerb durch „deed poll“ und der deutsche ordre public, StAZ 2016, 225 (229 ff.). 
Abs. 3 der Weimarer Reichsverfassung ${ }^{145}$ (WRV) sowie nach Art. 3 Abs. 1 GG (allgemeiner Gleichheitssatz, der standesrechtliche Unterscheidungen untersagt) und Art. 20 Abs. 1, 2, Art. 28 Abs. 1 S. 1 GG (Ablehnung der Monarchie, deren Wesenselement gerade Privilegien einzelner Personen aufgrund einer Familienzugehörigkeit sind) kennt das deutsche Recht keinen Adel mehr und damit auch keinen Scheinadel. Bei einer Reform des Namensrechts durch einen republikanischen Gesetzgeber dürften Adelsbezeichnungen daher namensrechtlich keine Exklusivität mehr genießen, um eine echte Gleichstellung im Namensrecht zu erreichen und Adelsbezeichnungen keinen Verwässerungsschutz zu gewähren, anders als das bisher der Fall war. Zwar hat Art. 109 Abs. 3 WRV Adelsprivilegien abgeschafft. Allerdings wurden die bestehenden Adelsbezeichnungen in Namensbestandteile umgewandelt und konnten über das dynastisch geprägte bürgerliche Namensrecht weitergegeben werden, weitgehend in den Bahnen der vorher für die Weitergabe dieser Prädikate geltenden Regeln. ${ }^{146}$ Das Reichsgericht hatte im Jahr 1926 sogar klargestellt, dass Adelsbezeichnungen an den jeweiligen Namensträger geschlechtsspezifisch angepasst und gebeugt werden, ${ }^{147}$ wobei der Bundesgerichtshof kürzlich auf Distanz zu dieser Praxis gegangen ist, die „den Eindruck einer Reminiszenz an früheres Adelsrecht entstehen [lässt] und deshalb dem eigentlich erwünschten gesellschaftlichen Bedeutungswandel bezüglich der Adelsbezeichnungen nicht förderlich [ist]“. ${ }^{148}$ Eine namensrechtliche Abschaffung des Adels wäre erst vollendet, wenn dessen ehemalige Bezeichnungen nicht mehr exklusiv sind und namensrechtlich das Motto „Adel für jedermann“ gilt. ${ }^{149}$ Zwar hat der Bundesgerichtshof für das geltende Namensrecht zu Recht darauf hingewiesen, dass die Möglichkeit, eine ehemalige Adelsbezeichnung nach Art. 48 EGBGB zu wählen, „kein[en] messbare[n] Einfluss auf den gesellschaftlichen Bedeutungswandel in Bezug auf Adelsbezeichnungen [...] erwarten“ lässt, da nur wenigen Personen dieser Weg offenstehen würde. ${ }^{150}$ Diese Überlegung greift jedoch nicht mehr, wenn die Namenswahlfreiheit zum Grundprinzip des deutschen Namensrechts würde.

145 Verfassung des Deutschen Reiches vom 11.8.1919, RGBl. 1919, S. 1383.

146 Siehe aber auch Baring Der Adel und sein Name im neuen Recht, FischersZ 51 (1920) 225 (256), der infolge der Anwendung des bürgerlichrechtlichen Namensrechts auf ehemalige Adelsbezeichnungen konstatiert: „Überall Scheinadel!“.

147 RG 10.3.1926, RGZ 113, 107.

148 BGH 14.11.2018, StAZ 2019, 77 = FamRZ 2019, 218, Rn. 34.

149 Dutta (Fn. 101) 1218 f.; gegen eine ordre-public-Widrigkeit von frei gewählten Adelsbezeichnungen auch Otto (Fn. 144) 229 ff.; ders. Von deutscher Namensrepublik. Neues zum Erwerb eines Adelsprädikates durch englische „deed poll“, StAZ 2019, 71.

150 BGH 14.11.2018, StAZ 2019, 77 = FamRZ 2019, 218, Rn. 34. 


\section{Oder doch: Keine umfassende Namenswahlfreiheit mit Grenzen, sondern eine beschränkte Namenswahlfreiheit aus anerkennenswerten Gründen?}

Mir ist durchaus bewusst, dass eine umfassende Namenswahlfreiheit des Einzelnen als Ausgangspunkt einer gesetzlichen Regelung ein Schritt sein könnte, der politisch zu groß ist, wenn man bedenkt, wo sich das deutsche Namensrecht derzeit befindet. Deshalb darf man - und das haben auch die Diskussionen in der Arbeitsgruppe des Bundesinnenministeriums und des Bundesjustizministeriums zur Reform des Namensrechts gezeigt - für jeden kleinen Schritt in die richtige Richtung dankbar sein. So könnte man - ähnlich wie das schweizerische Zivilgesetzbuch in Art. 30 Abs. 1, der eine Namensänderung aus „achtenswerten Gründen“ gestattet - die Namenswahlfreiheit auf anerkennenswerte Gründe reduzieren, also nicht negativ beschränken (alles ist erlaubt, was nicht verboten ist), sondern positiv definieren (alles ist verboten, was nicht erlaubt ist). Diesem Weg folgt im Grundsatz auch das Eckpunktepapier des Bundesinnenministeriums und des Bundesjustizministeriums. ${ }^{151}$

Allerdings ist eine solche Herangehensweise nicht ohne Schwierigkeiten, wie stets, wenn das Recht den Umfang der menschlichen Freiheit positiv für Fälle definieren will, in denen eine Ausübung der Freiheit dem Gesetzgeber sinnvoll erscheint. Unter welchen Voraussetzungen sollte eine Namensänderung zulässig sein? Nur bei einer Statusänderung (Eheschließung, Beendigung der Ehe, Vaterschaftsanfechtung, Vaterschaftsfeststellung etc.), nur bei der Änderung des Familiennamens einer Person, von der die Person ihren Namen ableitet, nur bei einer Änderung der sozial-familiären Beziehungen des Namensträgers, nur bei einer drohenden Beeinträchtigung des Wohls des Namensträgers durch anstößige oder negativ besetzte Namen, nur zur Namensintegration und zur Beseitigung einer hinkenden Namensführung? Soll man die freie Namenswahl als einen „Freischuss“ ausgestalten, als ein Recht, das in einem bestimmten Zeitraum nur einmal ausgeübt werden darf, etwa alle zehn Jahre? Jeder dieser positiven Voraussetzungen für eine Namenswahlfreiheit - die im Wesentlichen aus dem Eckpunktepapier des Bundesinnenministeriums und des Bundesjustizministeriums stammen $^{152}$ - wohnt etwas Willkürliches inne. Es wird einerseits immer Fälle geben, in denen eine Namenswahl aus Sicht der Selbstdarstellungsfunktion (oben V. 1.) legitim wäre, aber von den gesetzlichen Fallgruppen nicht erfasst sein wird. Andererseits sollte man Eltern im Einzelfall eine Namensänderung des Kindes aus

151 Eckpunkte zur Reform des Namensrechts (Fn. 2) S. 5 f. unter III. 4. 152 Eckpunkte zur Reform des Namensrechts (Fn. 2) S. 5 f. unter III. 4. 
Kindeswohlgründen verweigern können (vgl. zur Bedeutung der Namensstabilität für das Kindeswohl oben V. 2. a), wo starre Fallgruppen eine Namensänderung für das Kind ohne Weiteres zulassen würden.

Vielleicht findet sich deshalb doch der Mut zu einer im Grundsatz umfassenden Namenswahlfreiheit, die übrigens rechtsvergleichend keine Ausnahmeerscheinung mehr wäre, und zwar auch jenseits des common law, das bereits traditionell mit dem deed-poll-Verfahren eine freie Namensänderung ermöglicht (vgl. bereits oben IV. 5). Schweden und Dänemark, die beide eine grundsätzlich freie Namenswahl zulassen, wurden bereits erwähnt (oben V. 2. b). Eine ähnliche Namenswahlfreiheit besteht seit einigen Jahren auch in Slowenien, ${ }^{153}$ im Kosovo, ${ }^{154}$ in Kroatien, ${ }^{155}$ Nordmazedonien ${ }^{156}$ und in der Ukraine ${ }^{157}$. Auch unser Nachbarland Österreich hat vor einigen Jahren sein Namensrecht erheblich liberalisiert, konkret die Vorschriften zur Namensänderung, die jedem Bürger die Wahl eines „Wunschnamens“ gestatten. ${ }^{158}$ Verhaltenssteuernd wirken hier allein die Verwaltungsgebühren: Wer einen wichtigen Grund vorträgt, kann seinen Namen weitgehend gebührenlos ändern, die grundlose Namensänderung ist dagegen mit einer „Bewilligungsgebühr“ von derzeit 545,60 Euro verbunden. Die bisherigen Erfahrungen gerade in Österreich zeigen, dass ein Mehr an Freiheit im Namensrecht weder zu täglichen und unkontrollierten Namenswechseln der Bürger führt noch die Standesämter oder Gerichte vor unlösbare Fragen stellt. ${ }^{159}$ Die Bürger werden nicht leichtfertig von der Namenswahlfreiheit Gebrauch machen. Vor 45 Jahren wies bereits Alexander Blankenagel zu Recht darauf hin, dass jede „Namensänderung [...], wie nicht vergessen werden sollte, für den ,Änderer ein großes soziales Risiko“ birgt. ${ }^{160}$ Womöglich führt eine Namenswahlfreiheit sogar zu weniger Namensänderungen (oben V. 1. und V. 2. a).

$153 \S 18$ des Zakon o osebnem imenu.

154 Art. 10 ff. des Ligji për emrin personal.

155 Art. 6 ff. des Zakon o osobnom imenu.

156 Art. 5 ff. des Zakon za lichnoto ime.

157 Art. 295 des Tsyvil'nyy kodeks.

$158 \S 2$ Abs. 1 Nr. 11 (gegebenenfalls in Verbindung mit §2 Abs. 2) des österreichischen Namensänderungsgesetzes, wonach eine Namensänderung möglich ist, sofern der Antragsteller diese „aus sonstigen Gründen [...] wünscht“.

159 Siehe Konstanze Winkler Die Namensänderung in Österreich - Erfahrungen aus der Praxis nach der Liberalisierung des Namensrechts, FamRZ 2020, 570; zur Reform des österreichischen Namensrechts siehe auch Susanne Ferrari/Alexandra Richter Das österreichische Kindschafts- und Namensrechts-Änderungsgesetz 2013, FamRZ 2013, 1457.

160 Blankenagel (Fn. 108) 962. 


\section{Standort eines liberalisierten Namensrechts und zuständige Behörden}

Abschließend stellt sich noch die eher rechtsästhetische Frage nach dem Standort eines liberalisierten Namensrechts. Richtigerweise sollte das Namensrecht - wie auch das Eckpunktepapier des Bundesinnenministeriums und des Bundesjustizministeriums vorschlägt ${ }^{161}$ - in das allgemeine Personenrecht integriert werden, also in den Allgemeinen Teil des Bürgerlichen Gesetzbuchs. Hier sind nicht nur einige Paragraphenstellen frei, konkret die §§ 3-6 BGB oder die §§ 15-20 BGB. Auch gehört hier der Name systematisch hin, neben die Rechtsfähigkeit und künftig womöglich das Geschlecht des Menschen. ${ }^{162}$ Dieser Platz liegt umso mehr nahe, wenn man, wie hier vorgeschlagen, den Namen vom familienrechtlichen Status des Menschen und damit von seiner traditionellen Klassifizierungsfunktion löst und die Selbstdarstellungsfunktion als Teil der menschlichen Persönlichkeit in den Mittelpunkt des rechtlichen Namens rückt.

Die Verortung des Namensrechts allein im Bürgerlichen Gesetzbuch und dort im Allgemeinen Teil wäre ein Vorteil einer Namenswahlfreiheit, die das bestehende Namensrecht sicherlich übersichtlicher gestalten würde. Auch würde eine Namenswahlfreiheit die bisher gespaltenen Zuständigkeiten für das Namenswesen überflüssig machen: Das Bedürfnis für das Institut der öffentlichrechtlichen Namensänderung - die Grenzen zwischen öffentlichrechtlicher Namensänderung und bürgerlichrechtlichem Namenserwerb hat der Gesetzgeber ohnehin in den vergangenen Jahrzehnten verwischt ${ }^{163}$ - würde entfallen ${ }^{164}$ und der Gesetzgeber könnte das Namensrecht bundeseinheitlich den Standesbeamten anvertrauen. ${ }^{165}$

161 Eckpunkte zur Reform des Namensrechts (Fn. 2) S. 5 unter III. 1.

162 Referentenentwurf des Bundesjustizministeriums und des Bundesinnenministeriums zur Neuregelung der Änderung des Geschlechtseintrags vom 8.5.2019.

163 So bereits Benedikt von Schorlemer Die zivilrechtlichen Möglichkeiten der Namensänderung (1998) S. 161; so auch aus rechtsvergleichender Perspektive das Fazit von Hepting Entwicklung des Namensrechts im internationalen Vergleich, StAZ 2012, 257 (262).

164 So auch die Eckpunkte zur Reform des Namensrechts (Fn. 2) S. 5 unter III. 4.

165 So auch die Eckpunkte zur Reform des Namensrechts (Fn. 2) S. 5 unter III. 2 und S. 8 unter III. 10. Vgl. bereits Sturm (Fn. 3) 619; auch Bornhofen (Fn. 17) 230, forderte vom Gesetzgeber „eine konzertierte Aktion, die ein für beide Bereiche [gemeint ist das bürgerlichrechtliche Namensrecht und das öffentlichrechtliche Namensänderungsrecht, d. Verf.] einheitliches Regelwerk zum Ziel haben sollte“, die „der richtige Weg aus dem derzeitigen Namensdschungel“ wäre. 